\title{
Effect of Guide Vane Pitch Circle Diameter on Pressure Fluctuation in Vaneless Zone of Francis Turbine
}

\author{
Z. J. Wu${ }^{1}$, W. K. Liang $^{1 \dagger}$ and W. Dong ${ }^{2}$ \\ ${ }^{1}$ Institute of Water Resources and Hydro-electric Engineering, Xi'an University of Technology, Xi 'an \\ 710048, China \\ ${ }^{2}$ College of Water Resources and Architectural Engineering, Northwest A\&F University, Yangling, Shaanxi \\ 712100, China
}

†Corresponding Author Email: liangwuke@vip.163.com

(Received September 4, 2019; accepted February 12, 2020)

\begin{abstract}
In a Francis turbine, the guide vanes are arranged in the direction of flow behind the stay vanes. And as it is generally believed that the outlet angle of water after it flows through the spiral case and stay vanes is fixed, the flow and flow circulation are altered by changing the opening of the guide vanes so as to change the output of the turbine. The rotor-stator interaction effect induced by the interaction between the guide vanes and the runner of the Francis turbine was one of the main causes of the pressure fluctuation. The effect of guide vanes placement on pressure fluctuation in vaneless zone of Francis turbine was studied. In this study, the commercial software ANSYS CFX16.0 was used for the three-dimensional numerical simulation of the whole flow passage of a Francis turbine model in a power station. The turbulence model used in the calculation was the shear stress transport (SST) model. The independence between the total number of computational meshes and the timestep was verified to ensure the reliability of the calculation results. Five schemes with different diameters of the guide vanes distribution circle were proposed including $D_{0} / D_{1}$ (guide vane pitch circle diameter /diameter of runner inlet) equaling to $1.119,1.128,1.138,1.144$, and 1.15 . The steady calculation results showed that, when the turbine was operating under the design condition, $D_{0} / D_{1}$ increased from 1.119 to 1.15 , and the turbine efficiency and output showed a monotonically increasing trend, with the efficiency increased by 0.17 percentage point and output increased by $3.91 \mathrm{kw}$. Twenty monitoring points were set up in the vaneless zone between the guide vanes and the runner to collect pressure fluctuation signals in the vaneless zone. By analyzing the characteristics of unsteady pressure fluctuations in the vaneless zone under design conditions of the five schemes, the optimal position of the guide vanes was determined. The numerical results showed that the pressure fluctuation amplitude at monitoring points in the same axial direction increased gradually from the top cover to the bottom ring. When the unit operated under the design conditions, by increasing the guide vane pitch circle diameter, the rotor-stator interaction between the guide vanes and the runner domain was weakened, and the pressure fluctuation amplitude in the vaneless zone between the guide vanes and the runner was reduced, thereby the stability of unit operation was improved.
\end{abstract}

Keywords: Francis turbine; Guide vane; Pitch circle diameter; Vaneless zone; Pressure fluctuation.

\section{NOMENCLATURE}

\begin{tabular}{|c|c|c|c|}
\hline$C_{p}$ & pressure coefficient & $Q$ & flow rate \\
\hline$D_{0}$ & diameter of guide vanes & $Q_{11}$ & unit discharge of the model turbine \\
\hline$D_{1}$ & diameter of runner inlet & $\widetilde{Q}_{\mathrm{d}}$ & rated discharge \\
\hline$g$ & acceleration due to gravity & $t$ & time \\
\hline$f_{\mathrm{n}}$ & rotation frequency & $\mathrm{y}^{+}$ & non dimensional wall distance \\
\hline $\begin{array}{l}H \\
n\end{array}$ & $\begin{array}{l}\text { the head of a water turbine in operation } \\
\text { rotational speed of the runner }\end{array}$ & $x$ & sample value \\
\hline$N$ & the number of samples & $\rho$ & density \\
\hline$n_{11}$ & unit speed of the model turbine & $\sigma$ & sample data standard deviation \\
\hline $\bar{p}$ & force acting on the lower side & $\mu$ & the arithmetic mean of the sample \\
\hline
\end{tabular}




\section{INTRODUCTION}

Hydropower is one kind of renewable and clean energy resources, and thus being an important part of power generation in many countries (Giosio et al. 2017). Turbines convert hydropower into mechanical energy to drive generators and generate electricity.

The stability of turbine operation is directly related to the safety of power station (Gavrilov et al. 2016; Romero-Gomez et al. 2017). The vibration generated during the operation of the unit mainly comes from two reasons: mechanical vibration caused by manufacturing and installation errors and hydraulic vibration caused by unstable internal flow of the unit. The damage caused by hydraulic vibration is very great. In the Sayano-Shushenskaya Hydro Power Plant in Russia, accidents occurred because the pressure pulsation was too large, the unit vibration exceeded the standard, and the roof fastening bolt fatigue fracture caused the water flooding of the plant (Peltier et al. 2011; Bellendir et al. 2011).

Pressure fluctuation is the inherent cause of hydraulic vibration (Muller et al. 2012). The pressure fluctuation in the turbine runner mainly includes the pressure fluctuation in the vaneless zone, the pressure fluctuation in the runner area, the pressure fluctuation in the draft tube, and the pressure fluctuation generated by the blade vortex and the karmen vortex (Trivedi et al. 2017). Among them, the pressure pulse action in the vaneless zone is one of the main sources of hydraulic instability of the water wheel, and it has been a research hotspot.

Ciocan et al. (2007) analyzed the velocity field distribution in the vaneless zone in detail through LDV and PIV, and obtained a velocity pulsation cloud map between the flow channels of active guide vanes at different times. Staubli et al. (2008) used numerical simulation to analyze the vortex and backflow phenomena in the vaneless zone and the flow and torque fluctuations caused by the pump turbine in the "S" area, and found that the local vortex structure at the inlet of the runner passage was the source of the unsteady flow in the bladeless area, and most of the energy dissipation was concentrated in the bladeless area.

The pressure fluctuation in the vaneless zone is mainly caused by the dynamic and static interference between the moving guide vane and the runner. As an important flow passage component of the turbine, the main function of the guide vanes is to regulate the flow running through the turbine and provide circulation for the runner inlet (Koirala et al. 2017). The outlet angle of the guide vanes directly affects the angle of attack of the water impinging on the runner blade, which in turn affects the runner's work efficiency (Pereiras et al. 2014; Thapa et al. 2016; Tyagi et al. 2015). The structure of guide vanes has been studied and optimized by many researchers. Li et al. (2011) optimized the turbine guide vanes under two-phase flow conditions, the position of the guide vanes, as well as the matching relationship between the stay van es and guide vanes. In addition, the loss of water head in the guide vanes was reduced, so as to improve the efficiency of the runner. Koirala et al. (2016) numerically calculated the internal flow field of a Francis turbine. It was found that by increasing the end clearance of the guide vanes, the amount of flow that leaked through the clearance increased, and the unit efficiency was reduced. Steady pressure fluctuations could enhance the vibration and swing of the unit as well as the dynamic stress of the runner blade. The degree of influence mainly depended on the magnitude of the pressure fluctuation and the operating range.

At present, numerical simulation technology has been widely used in the prediction of turbine pressure fluctuations (Iovănel et al. 2019). Dynamic mesh methodology was used for simulations in which GVO (Guide Vane Opening) of a pumpturbine from $9 \mathrm{~mm}$ to $26 \mathrm{~mm}$ by Han et al. (2017). Yan et al. (2010) investigated the influence of water compressibility on pressure fluctuations induced by rotor-stator interaction (RSI) in hydraulic machinery, using the commercial CFD solver ANSYS-CFX. Compared with the experimental results, incompressible simulations of RSI could predict pressure fluctuations in vaneless space. Minakov et al. (2015) used the detached eddy simulation (DES) turbulence model and "frozen rotor" method to simulate unsteady turbulence of a Francis turbine with high water head. Combined with test data, it was proved that the numerical method could be used to accurately predict pressure fluctuation characteristics of a unit under different operating conditions. The main frequency and intensity of the pressure fluctuation in the runner could be obtained, and the accuracy could reach $10 \%$. At present, research on the influence of guide vanes on pressure fluctuations in the vaneless zone mainly focuses on the characteristics of pressure fluctuations in the runner when the internal flow pattern of the turbine changes dramatically under working or non-working conditions. By means of a numerical method (Trivedi et al. 2016), it was found that the amplitude of the pressure fluctuations in the vaneless zone during load rejection was approximately 2.8 times the amplitude under optimal operating conditions.

In this study, a computational fluid dynamics (CFD) method was used. For a certain Francis turbine prototype, the pressure fluctuation was analyzed in the vaneless zone between the guide vanes and the runner under the design condition, and the relative variations in pressure fluctuations were compared for guide vanes distribution circles with five different diameters. By analyzing the components and amplitudes of the pressure fluctuations at the monitoring points in the channel, the influence on turbine stability caused by the diameter of the distribution circle was analyzed to determine the optimal position of the guide vanes in the radial direction.

\section{Study ObJect ANd Modelling}

\subsection{Computational Model and Design Parameters}

The prototype turbine is similar to the model turbine, 


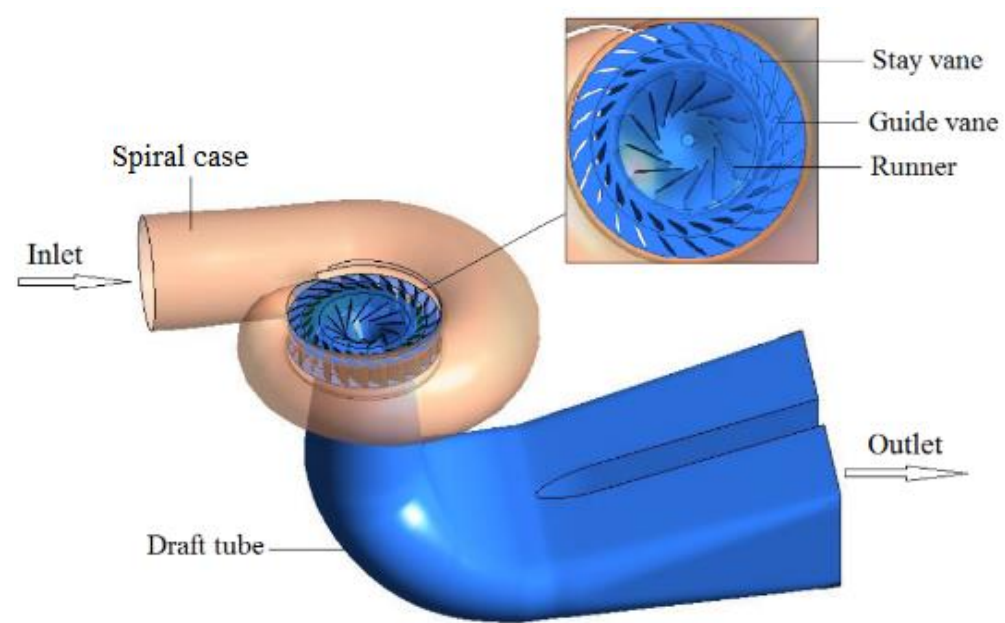

Fig. 1. 3D whole computational mode of Francis hydro-turbine.

the unit speed $n_{11}$ and unit flow $Q_{11}$ are defined as follows:

$$
\begin{aligned}
& n_{11}=\frac{n \cdot D_{1}}{\sqrt{H}} \\
& Q_{11}=\frac{Q}{D_{1}^{2} \sqrt{H}}
\end{aligned}
$$

Where $n$ is the rotational speed of the runner, the unit is $\mathrm{r} / \mathrm{min} ; Q$ is the flow rate, the unit is $\mathrm{m}^{3} / \mathrm{s} ; D_{1}$ is the diameter of the runner inlet, the unit is $\mathrm{m} ; H$ is the head of a water turbine in operation, the unit is $m$.

Taking the Francis turbine model of a power station as the study object, the numerical calculation was carried out. The results were as follows: the head was $H=30 \mathrm{~m}$; the design unit speed of the prototype was $n_{11}=69 \mathrm{r} / \mathrm{min}$; the unit discharge was $Q_{11}=0.765 \mathrm{~m}^{3} / \mathrm{s}$; and the guide vane opening was $12.8 \mathrm{~mm}$. The entire calculation area included the spiral case, stay vanes, guide vanes, runner, and draft tube. The main parameters of the flow passage components are shown in Table 1. Unigraphics NX was used for geometric modeling, as shown in Fig. 1.

Table 1 Specification parameters of flow passage components

\begin{tabular}{|c|c|c|}
\hline Domain & Parameters & Values \\
\hline Stay vanes & Number of stay vanes & 24 \\
\hline \multirow{2}{*}{$\begin{array}{c}\text { Guide } \\
\text { vanes }\end{array}$} & Number of guide vanes & 24 \\
\cline { 2 - 3 } & Guide vane height & $0.27 D_{1}$ \\
\hline \multirow{2}{*}{ Runner } & $\begin{array}{c}\text { Distribution circle } \\
\text { diameter (mm) }\end{array}$ & 409.8 \\
\cline { 2 - 3 } & $\begin{array}{c}\text { Number of runner blades } \\
\text { Runner inlet diameter } D_{1} \\
(\mathrm{~mm})\end{array}$ & 13 \\
\hline
\end{tabular}

\subsection{Numerical Computation}

The internal flow of a turbine falls within the category of incompressible turbulent flow, and it follows the law of conservation of mass and the law of conservation of momentum. The convergence speed and calculation accuracy of different turbulence models are different. In the shear stress transport (SST) model, the $k-\varepsilon$ turbulence model is combined with the Wilcox two-equation $k-\omega$ turbulence model through a blending function (Menter et al. 1994). The model $k$ - $\omega$ is used near the wall, and the $k-\varepsilon$ model is used in the well-developed flow field away from the wall (Anup et al. 2014; Campobasso et al. 2018). Thus, the SST model fully combines the high precision of the $k-\omega$ model in separated flow simulations and the low sensitivity of the $k-\varepsilon$ model to initial turbulence parameters, making it one of the most widely used turbulence models at present. In this study, CFD commercial software ANSYS CFX16.0 was used for the numerical simulation, and the SST model was used.

The inlet boundary condition of mass flow was used for the spiral case inlet, and the pressure outlet boundary condition was adopted for the draft tube outlet. Mean static pressure was applied, and the hydrodynamically smooth and non-slip condition was adopted for the wall boundary. In the steady calculation, "Frozen-Rotor" was used for the dynamic-static interface of the inlet and outlet in the runner area, and the convergence criterion for the calculations was set to a maximum residual less than $10^{-4}$. After the steady calculation was completed, the results of it were used as initial values for the calculation of unsteady pressure fluctuations. In the calculation, the dynamic-static interfaces between the guide vanes and the runner, and between the runner and the draft tube, were set as the "Transient Rotor-Stator." In this model, the relative position relationship between the dynamic and static domain with different timesteps were taken into account, so it could truly predict the transient impact at the dynamic and static boundaries (Lain, 2018).

\subsection{Meshing and Test for Independence}

ICEM CFD(The Integrated Computer Engineering and Manufacturing code for Computational Fluid Dynamics) was used to generate high-quality structured meshes for the flow passage components of the turbine and to refine the meshes at certain important walls in the flow field in order to capture more detailed flow field information. The computational domain meshes of the flow passage components are shown in Fig. 2. In order to ensure the accuracy of the calculation results, an 
independence test was performed on the meshes. As shown in Table 2, with the efficiency and output of the original turbine unit under design conditions as reference indicators, and When the number of grids increased from 5.5 to 7.5 million, turbine efficiency increased by only 0.01 percentage point, indicating that 5.5 million was an independent solution of the grid number. Therefore, the total number of meshes for the numerical calculation of the whole flow passage in the model turbine was determined to be 5.5 million. Table 3 shows the grid characteristics of the flow passage components in the computational domain when the total grid number is 5.5 million.
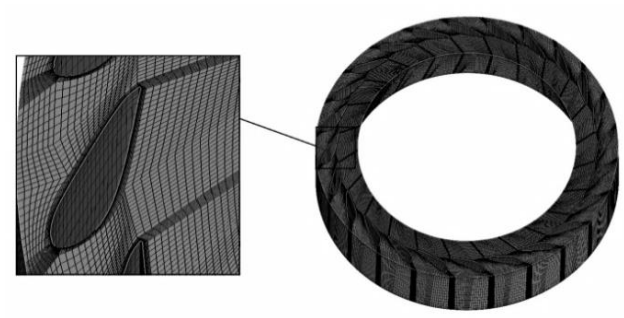

a)
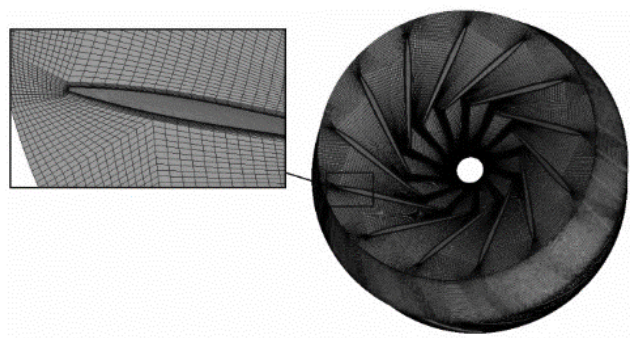

b)

Fig. 2. Grid generation of guide vanes and runner. a) Guide vanes b) Runner.

Table 2 Grids independence test and verify

\begin{tabular}{|c|c|c|c|}
\hline Plan & $\begin{array}{c}\text { Total } \\
\text { elements } \\
\left.10^{4}\right)\end{array}$ & $\begin{array}{c}\text { Efficiency } \\
(\%)\end{array}$ & $\begin{array}{c}\text { Power } \\
(\mathrm{kW})\end{array}$ \\
\hline $\mathrm{A}$ & 350 & 94.16 & 150.13 \\
\hline $\mathrm{B}$ & 550 & 94.28 & 150.24 \\
\hline $\mathrm{C}$ & 750 & 94.27 & 150.27 \\
\hline
\end{tabular}

Table 3 Mesh Description

\begin{tabular}{|c|c|c|c|c|}
\hline Domain & $\begin{array}{c}\text { Total } \\
\text { elements }\end{array}$ & Total nodes & $\begin{array}{c}\text { Min } \\
\text { angle }\end{array}$ & $\mathrm{y}+$ \\
\hline Spiral case & 537,352 & 558,946 & 38 & 402 \\
\hline Stay vanes & 803,564 & 877,382 & 45 & 54 \\
\hline $\begin{array}{c}\text { Guide } \\
\text { vanes }\end{array}$ & $1,816,128$ & $1,944,266$ & 48 & 43 \\
\hline Runner & $1,314,288$ & $1,394,558$ & 30 & 25 \\
\hline Draft tube & $1,043,478$ & $1,070,615$ & 47 & 229 \\
\hline
\end{tabular}

\section{Experimental Verification}

In order to verify the reliability of the numerical calculation, a real-machine efficiency test was carried out on the studied turbine during the clear flow period in winter, and the results were compared with those of CFD analysis. Both the inlet pressure of the spiral case and the pressure fluctuation of the draft were measured by using PR-21Y pressure sensors which were manufactured by Keller, Swiss. The accuracy of the pressure sensor was $\pm 0.2 \%$, and the response time was less than $0.5 \mathrm{~ms}$. The flow of the unit was recorded using an UF-911 ultrasonic flowmeter manufactured by Nari Group Corporation, Nanjing, China. The accuracy of the flowmeter was $\pm 0.5 \%$, and it was calibrated on site. During the experiment, the unit speed was $n_{11}=69 \mathrm{r} / \mathrm{min}$, and the unit flow was set to $0.8 Q_{\mathrm{d}}, 0.9 Q_{\mathrm{d}}, Q_{\mathrm{d}}, 1.1 Q_{\mathrm{d}}$, and 1.2 $Q_{\text {d. }}$

Fig. 3a) shows the efficiency comparison between the numerical calculation results and the model test results. As shown in Fig. 3a), the change rule of the calculated value was the same as that of the test value. When the unit flow rate was $Q_{\mathrm{d}}$, the inflection point of the efficiency value appeared, which was the highest point of the efficiency value. Because of the errors in machining of the flow passage components and the fact that the loss along the channel was not taken into account in the numerical calculation, the numerically calculated efficiency was slightly higher than the test efficiency. The maximum error between the calculated value and the test value was $0.8 \%$, which occurred when the unit flow was $0.9 Q_{\mathrm{d}}$. Overall, the hydraulic efficiency of the model turbine calculated using the numerical method was in good agreement with the test results, and the error was within an acceptable range.

The pressure value obtained by the numerical calculation was processed using a dimensionless method. The dimensionless fluctuation coefficient $C_{p}$ was used to describe the magnitude of the pressure fluctuation. The expression of the pressure fluctuation coefficient is as follows:

$C_{p}(i)=\frac{p(i)-\bar{p}}{\rho g H} \times 100 \%$

where $p(i)$ denotes the pressure at the monitoring point obtained by the numerical calculation, and the unit is $\mathrm{Pa} ; \bar{p}$ denotes the mean pressure at the monitoring point in a cycle obtained by the numerical calculation, and the unit is $\mathrm{Pa}$; and $\rho, g$, and $H$ denote the density of water, the local gravitational acceleration, and the calculated water head, respectively, and the units are $\mathrm{kg} / \mathrm{m}^{3}, \mathrm{~m} / \mathrm{s}^{2}$, and $\mathrm{m}$, respectively.

Fig. 3b) shows the pressure fluctuation of the draft comparison between the numerical calculation results and the model test results. The monitoring points was in the elbow bend of the draft. As being shown in the figure, the pressure fluctuation obtained by numerical calculation was consistent with the test results. The pulsation amplitude and main pulsation frequency of the kind of results were basically consistent, which proved that the main characteristics of pressure fluctuation could be captured by the numerical calculation results. 


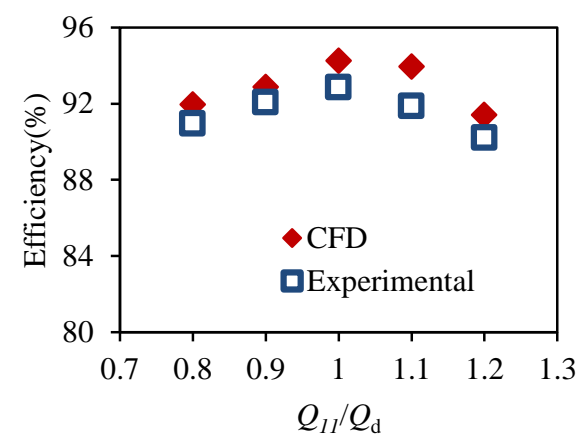

a)
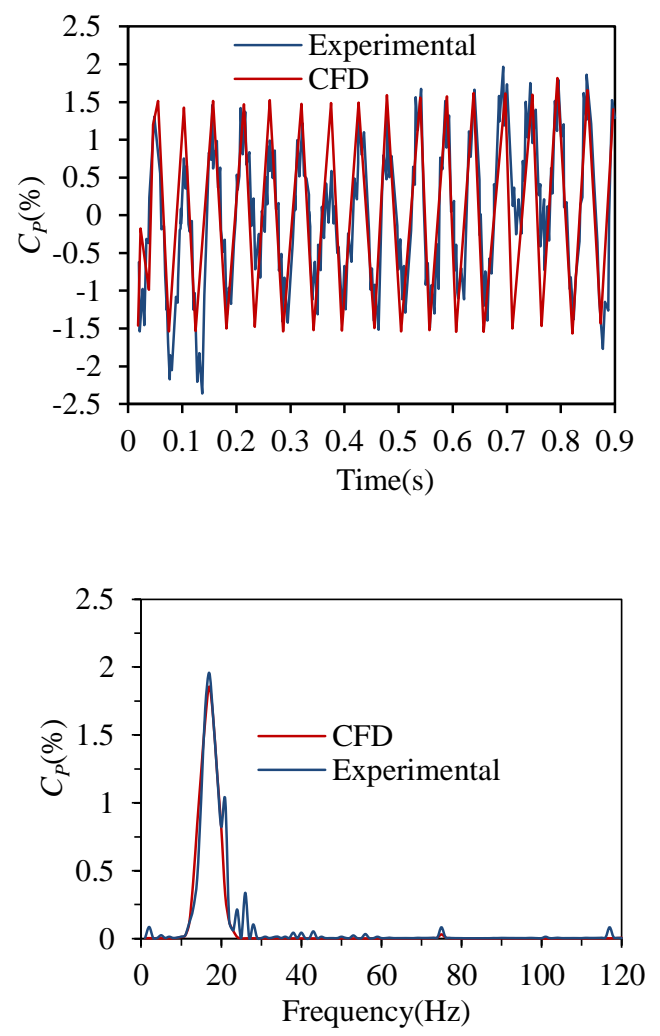

b)

Fig. 3. Comparisons between turbine efficiency experiment and numerical calculation: a)

Efficiency of the turbine. b) Pressure fluctuation of the draft.

\subsection{Monitoring Points Setup}

To study the effect of $D_{0}$ on the pressure fluctuation in the vaneless zone between the guide vanes and the runner, four groups of monitoring points were set up in the vaneless zone, each differing in 90 degrees in the circumferential direction, as shown in Fig. 4a). Each group of monitoring points consisted of five monitoring points distributed equidistantly from the top cover to the bottom ring. In each group, one monitoring point near the bottom ring was in the rotating region of the runner, and the other four were in the static region of the guide vanes, as shown in Fig. 4b). In total, there were 20 monitoring points in the vaneless zone.

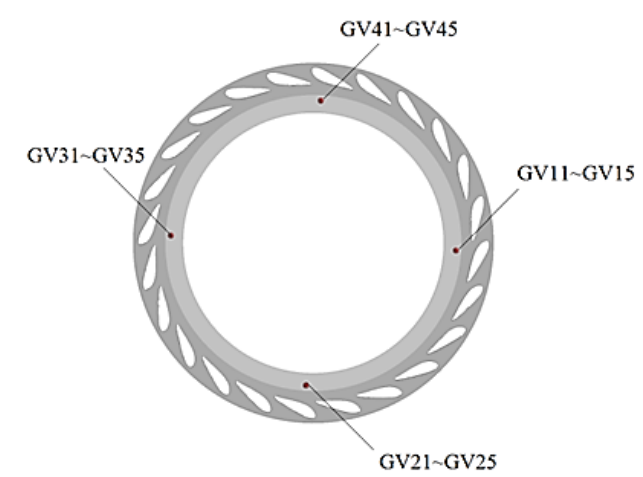

a)

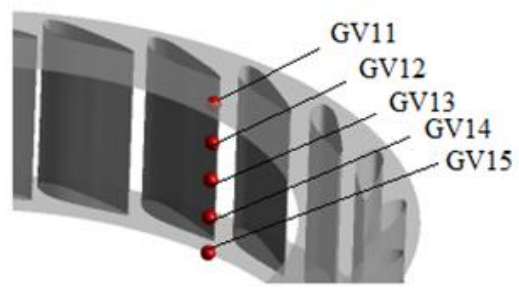

b)

Fig. 4. Monitoring points of vaneless zone: a) Arrangement of monitoring points in the vaneless zone around the circle. b) Arrangement of the first group of monitoring points.

\subsection{Timestep Independence Test}

The accuracy and stability of the numerical calculation were significantly influenced by the timestep. Non-convergence was likely to occur when the timestep was too large. An excessive timestep meant a lower sampling frequency during the pressure fluctuation calculation. If the sampling frequency was less than twice the maximum frequency component, the original signal of the pressure fluctuation could not be accurately reproduced, resulting in an inaccurate pressure fluctuation prediction. Therefore, the timestep needed to be small enough to obtain a sufficient frequency resolution. However, if the timestep was too small, it would cause unnecessary computation and take more time. In this study, the influences of four timesteps on the pressure fluctuation calculation were compared, and a total of 5.5 million structured meshes were used for the calculation. In the numerical calculation, the operating condition of the turbine was the design condition, and the speed was $69 \mathrm{r} / \mathrm{min}$. The time required for each rotating cycle of the runner was $0.057 \mathrm{~s}$. The timesteps used in the timestep independence test were $3.17 \times 10^{-4} \mathrm{~s}$, $3.97 \times 10^{-4} \mathrm{~s}, \quad 4.76 \times 10^{-4} \mathrm{~s}$, and $5.56 \times 10^{-4} \mathrm{~s}$, corresponding to rotations of $2 \mathrm{deg}, 2.5 \mathrm{deg}, 3 \mathrm{deg}$, and $3.5 \mathrm{deg}$, respectively. The corresponding sampling frequencies were $3150 \mathrm{~Hz}, 2520 \mathrm{~Hz}, 2100$ $\mathrm{Hz}$, and $1800 \mathrm{~Hz}$, respectively. Monitoring points GV11 and GV12 were studied to validate the pressure fluctuation in the vaneless zone.

The time-domain plots of the pressure fluctuation at monitoring points GV11 and GV12 with different timesteps in a rotating cycle are shown in Figs. 5-6. 
It can be seen that with the four timesteps, the pressure fluctuation waveforms at the same monitoring point were close, and the influence of timesteps on pressure fluctuations at the same monitoring point reflected the same rule. The pressure value of the timestep corresponding to 3.5 deg at the wave crest was obviously higher than the calculated values corresponding to $3 \mathrm{deg}, 2.5 \mathrm{deg}$, and $2 \mathrm{deg}$. The differences in the calculation results for the four timesteps are more clearly shown in the locally enlarged plots (Fig. 5b) and 6b)). It can be seen that the pressure waveforms of timesteps corresponding to $3 \mathrm{deg}, 2.5 \mathrm{deg}$, and $2 \mathrm{deg}$ were basically the same, indicating a high degree of consistency; these were significantly different from the waveform associated with the timestep corresponding to $3.5 \mathrm{deg}$. Therefore, with the calculation accuracy and the computation load taken into consideration, the timestep corresponding to $3 \mathrm{deg}$ was the independent solution to this timestep independence test. Hence, the timestep corresponding to $3 \mathrm{deg}$ (i.e., $4.76 \times 10^{-4}$ s) was selected for the numerical calculation of the pressure fluctuation in the vaneless zone.

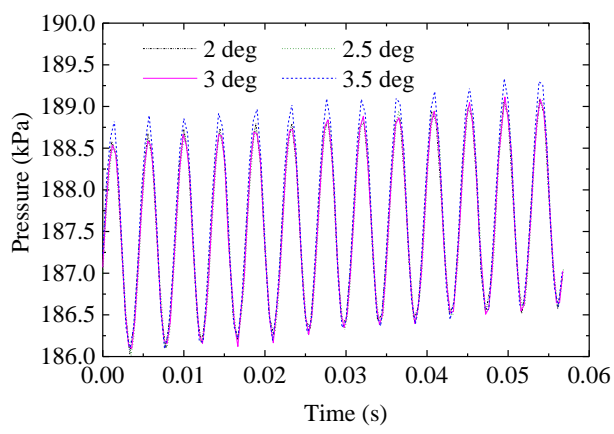

a)

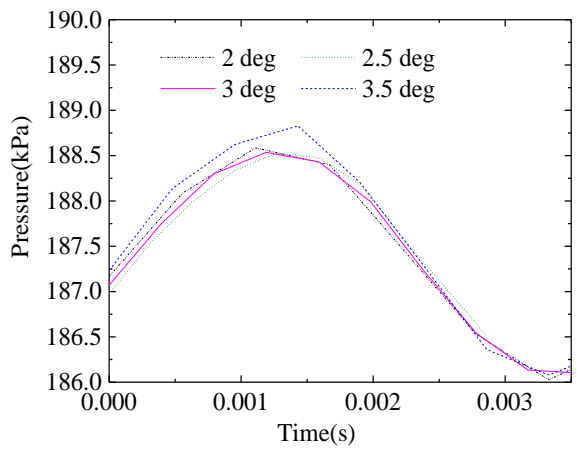

b)

Fig. 5. Timestep independence test at point GV11: Timestep independence test at point GV11. a) Time-domain plot of point GV11. b) Locally enlarged time-domain plot of point GV11.

\section{ModifiCATION}

The guide vanes of the Francis turbine and the axial flow passage of the runner are shown in Fig. 7, in which $D_{0}$ is the guide vane pitch circle diameter, and
$D_{1}$ is the diameter of the runner. To ensure that the guide vanes did not collide with the stay vanes or the runner at the maximum opening, the guide vanes were lapped reasonably when closed to ensure sealing, and the allowable variation range of the guide vane pitch circle diameter was $402-415.8 \mathrm{~mm}$. To analyze the influence of the distribution circle diameter on the performance of the turbine, five different schemes with different distribution circle diameters were put forward. Based on the original distribution circle of the guide vanes $\left(D_{0}=409.8\right.$ $\mathrm{mm})$, case a $\left(D_{0}=403 \mathrm{~mm}\right)$ and case $\mathrm{b}\left(D_{0}=406 \mathrm{~mm}\right)$ were obtained by reducing the diameter of the distribution circle, and case $\mathrm{d}\left(D_{0}=412 \mathrm{~mm}\right)$ and case e $\left(D_{0}=414 \mathrm{~mm}\right)$ were obtained by enlarging the diameter. The parameters of the schemes are shown in Table 4.

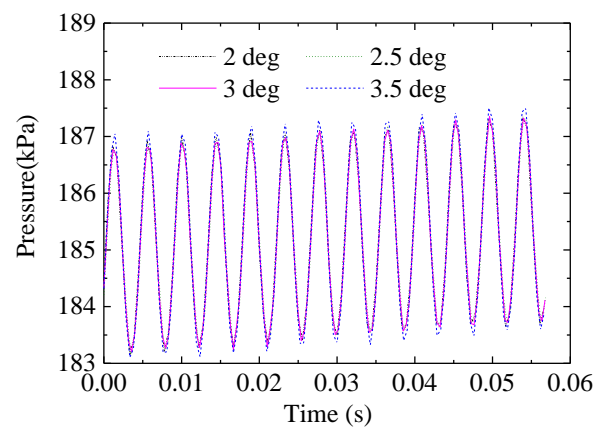

a)

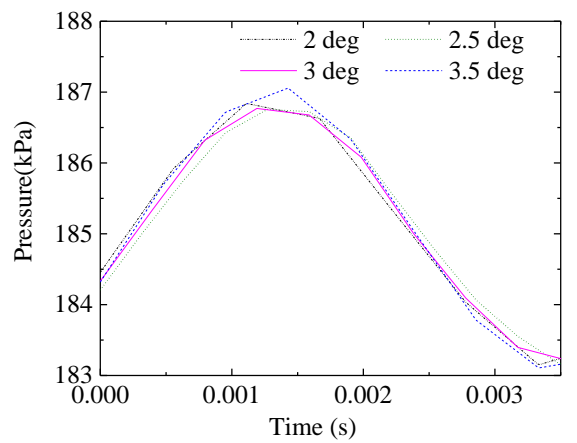

b)

Fig. 6. Timestep independence test at point GV12: Timestep independence test at point GV12. a) Time-domain plot of point GV12. b) Locally enlarged time-domain plot of point GV12.

\section{RESUlTS AND DISCUSSION}

\subsection{External Characteristic Analysis}

The runner is the core component of the turbine, and usually the head loss in the rotor accounts for the largest proportion of the total head loss. When the $\beta_{1}$ (inlet water flow angle of the runner, which is the angle between the relative velocity $\mathrm{w}_{1}$ and the peripheral velocity $u_{1}$ )is equal to $\beta_{\mathrm{b} 1}$ (the placement angle of the blade inlet), water flows smoothly around the blade, and the hydraulic loss is minimal. 


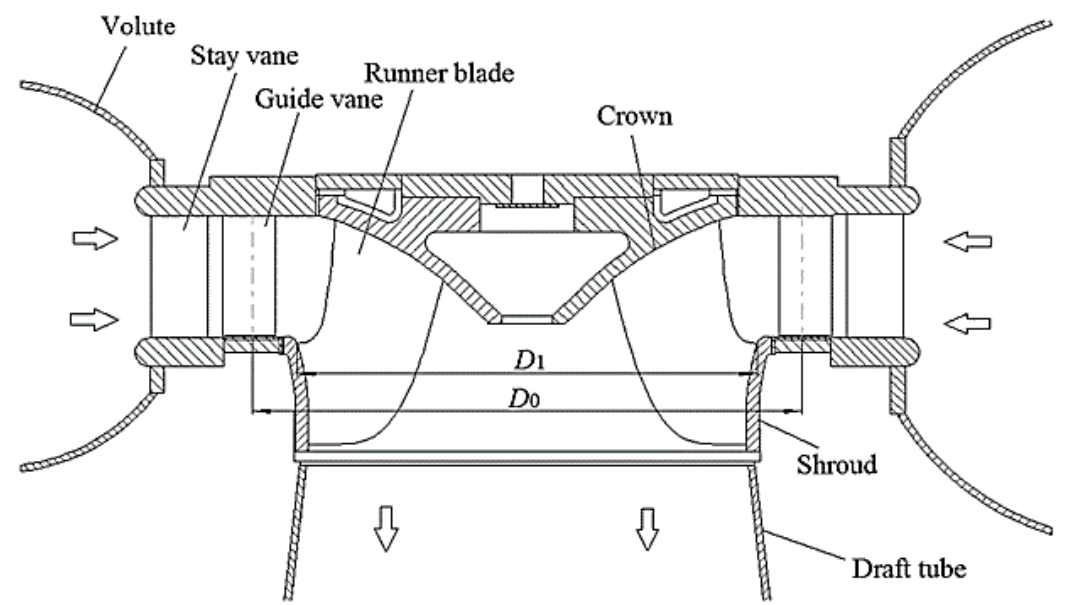

Fig. 7. Guide vanes distribution circle.

Therefore, the bigger the water flow angle of impact $\alpha\left(\alpha=\beta_{1}-\beta_{\mathrm{b} 1}\right)$ is, the greater the hydraulic loss is. Figure 8 is the speed triangle diagram at the inlet of the runner blade. Under the condition that the rotating angle of the active guide vane remains unchanged, the diameter of the distribution circle of the active guide vane is increased, the flow velocity $v_{1}$ is decreased, $\beta_{1}{ }^{\prime}<\beta_{1}$, and the hydraulic loss of the runner is decreased, thus improving the turbine efficiency.

Table 4 Diameter parameters of the guide vanes of each scheme

\begin{tabular}{|c|c|c|}
\hline \multirow{2}{*}{ Cases } & \multicolumn{2}{|c|}{ Parameters } \\
\cline { 2 - 3 } & $D_{0}(\mathrm{~mm})$ & $D_{0} / D_{1}$ \\
\hline $\mathrm{a}$ & 403 & 1.119 \\
\hline $\mathrm{b}$ & 406 & 1.128 \\
\hline $\mathrm{c}$ & 409.8 & 1.138 \\
\hline $\mathrm{d}$ & 412 & 1.144 \\
\hline $\mathrm{e}$ & 414 & 1.15 \\
\hline
\end{tabular}

Figure 9 shows the turbine efficiency and output power under different guide vanes distribution circle diameters. The results show that the turbine efficiency and output power have the same changing trend with the increase of the guide vane pitch circle diameter. In scheme a, the turbine efficiency and output are the lowest in the five schemes, with an efficiency value of $94.15 \%$ and an output value of $148 \mathrm{~kW}$.Scheme e has the highest efficiency and output, with an efficiency of $94.32 \%$ and output power of $151.9 \mathrm{kw}$. The turbine efficiency increases monotonically with the increase of the guide vane pitch circle diameter, and the efficiency increases gradually with the increase of $D_{0}$. Compared with case $a$, case $b$ increases turbine efficiency by 0.07 percentage point and output power by $0.84 \mathrm{kw}$, while case $e$ increases turbine efficiency by 0.02 percentage point and output by $0.78 \mathrm{kw}$ compared with case $d$. The difference between maximum and minimum efficiency of the five schemes is $0.17 \%$, and the difference between maximum and minimum output power is $3.91 \mathrm{kw}$.

(a)
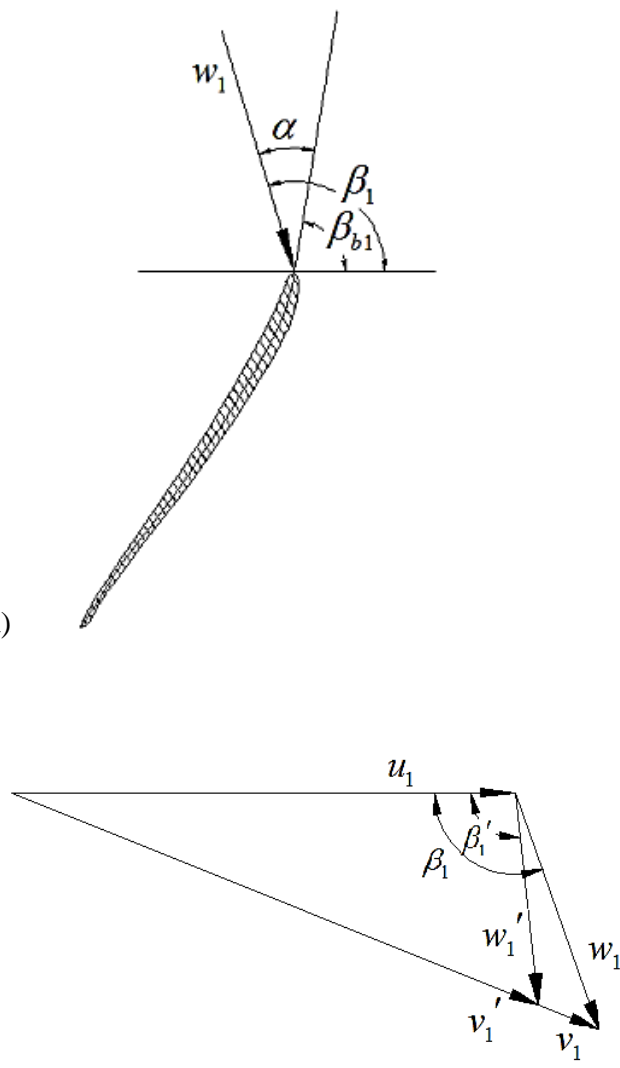

b)

Fig. 8. Attack angle and velocity triangle at the inlet of the runner blade. a) Attack angle diagram. b) The velocity triangle at the inlet of the runner blade.

\subsection{Pressure Fluctuation Analysis}

The rotor-stator interaction of hydro-turbine refers to the interaction between the rotating system (runner) and the stationary system (guide vane). 


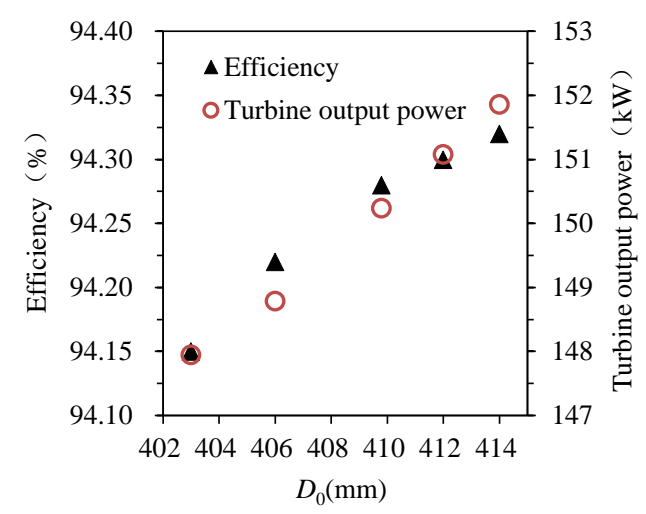

Fig. 9. Turbine efficiency and output power at different $D_{0}$.

The rotor-stator interaction effect induced by the interaction between the guide vanes and the runner of the Francis turbine was one of the main causes of the pressure fluctuation. The flow field in the vaneless zone between the guide vanes and the runner was mainly affected by the potential flow interference and the wake of the guide vanes. Potential flow interference was mainly caused by the interaction between the flow at the outlet of the guide vanes and the flow at the inlet of the runner blade. With the rotation of the runner, the velocity field and pressure field of the two superposed, causing pressure fluctuations in the vaneless zone. Phenomena such as Karman vortex and flow separation in the wake region of the outlet of the guide vanes could also disturb the flow field and cause pressure fluctuations in the vaneless zone. Rotor-stator interaction was induced by the interaction between the potential flow disturbance caused by the runner blade and the flow field disturbance, which was caused by the wake flow of the guide vanes. The change of $D_{0}$ would affect the flow at the outlet of the guide vanes, so it was necessary to study the influence of the changes in the guide vane pitch circle diameter on the pressure fluctuation in the vaneless zone.

Because the geometry of model turbine and prototype turbine is similar, when both of them are operating under the design condition, the outlet angle of guide vane and the inflow angle of runner are equal, the inlet flow of runner has a little angle of attack, and the outlet is close to normal outflow. According to the principle of similarity, it is considered that the flow field in vaneless area of model turbine is similar to prototype turbine.

After calculation, it was found that changes of the pressure fluctuation at the monitoring points in the same horizontal plane in the vaneless zone were insignificant in the circumferential direction. In the scheme of the original guide vane pitch circle diameter, the calculation results showed that the pressure fluctuation of the same group of monitoring points varied greatly in the axial direction (from the top cover to the bottom ring of the guide vanes). In this study, monitoring points GV11-GV15 were taken as examples to analyze the pressure fluctuation at monitoring points with five diameters of the guide vanes distribution circle.
Figures 10-14 shows the time-domain and frequency-domain plots of the pressure fluctuation at monitoring points GV11-GV15 for five $D_{0}$ schemes under the design flow conditions. The $f_{\mathrm{n}}$ denotes the blade passing frequency of turbine runner. As shown, under different $D_{0}$ schemes, the pressure fluctuations at the same monitoring point showed the same characteristics. In addition, it can be seen from the time-domain plot of five monitoring points that the pressure fluctuation at each monitoring point changed periodically. In the range of $t=0-0.12 \mathrm{~s}$, the waveforms of monitoring points GV11, GV12, and GV13 were the same. The time required for the runner to make one rotation was $0.057 \mathrm{~s}$, and the time interval between two adjacent peaks was $0.00428 \mathrm{~s}$. At this time, the runner rotated about $27 \mathrm{deg}$, which is, one runner blade cycle, indicating that the pressure fluctuation of the monitoring point was mainly interfered by the runner blade. The main frequency of the pressure fluctuation of monitoring points GV11, GV12, GV13, and GV14 was $13 f_{\mathrm{n}}$, which is the passing frequency of the runner blades. The pressure fluctuation of this part was mainly caused by the impact of the runner blades on the inlet water flow. The monitoring point GV14 was located near the rotating domain of the runner, and the pressure fluctuation was caused by the superposition of the amplitudes of different frequency components. The monitoring point GV15 was located near the bottom ring in the rotating runner domain, so it was not a stationary monitoring point. The pressure fluctuation received by GV15 was essentially the superposition of two uneven flow fields of the movable vane path and the runner path. The time interval between two adjacent peaks was $0.00238 \mathrm{~s}$, and the main frequency of monitoring point GV15 was approximately $24 f_{n}$, which was the combined frequency of the guide vanes and the runner blade.

Figure 15 shows the peak-to-peak pressure fluctuation values of monitoring points GV11 through GV15 under five diameters for the guide vanes distribution circle. The abscissa indicates the five monitoring points along the top cover to the bottom ring in the vaneless zone, and the ordinate indicates the dimensionless pressure fluctuation coefficient value of the peak-to-peak pressure fluctuation value. As shown in Fig. 15, the monitoring points of the vaneless zone are distributed from the top cover to the bottom ring. From the top cover to halfway down the guide vanes, the five peak-to-peak curves overlapped well, and the peak-to-peak value of the pressure fluctuation at each monitoring point gradually increased in a relatively stable way. From the $1 / 2$ height of the guide vanes to the bottom ring, the peakto-peak value of the pressure fluctuation at each monitoring point increased greatly, among which the maximum amplitude was obtained under the $D_{0}=403$ $\mathrm{mm}$ scheme, and the pressure fluctuation coefficient from monitoring point GV13 to monitoring point GV14 increased by $3.47 \%$. The guide vane pitch circle diameter mainly influenced the peak-to-peak value of the pressure fluctuation at monitoring point GV15, and the maximum difference between peaks was $1.69 \%$. As shown in Fig. 13, the peak-to-peak value of each monitoring point was the lowest under the scheme of $D_{0}=414 \mathrm{~mm}$. 


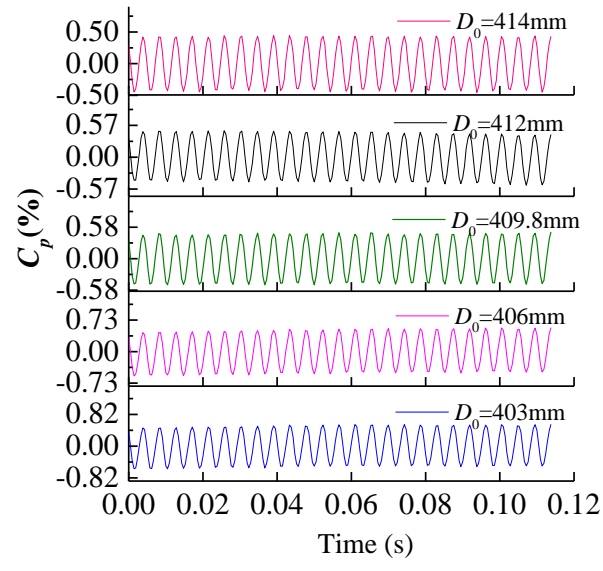

a)

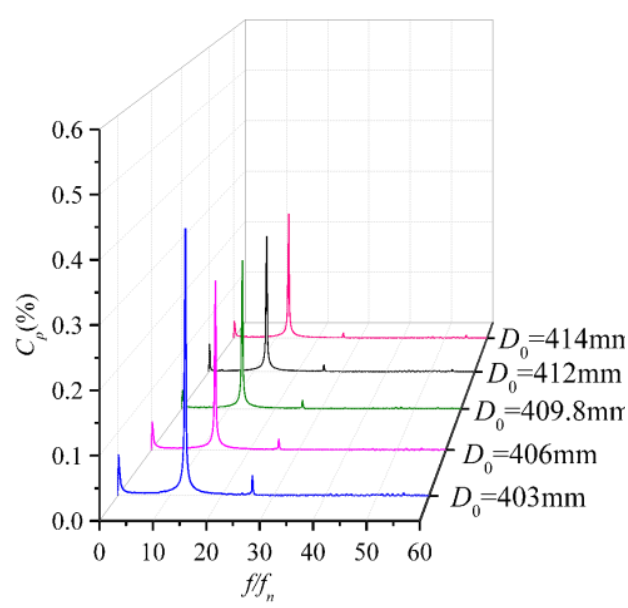

b)

Fig. 10. Plots of time-domain and frequency-domain pressure fluctuation of monitoring points GV11: a) Plots of time-domain pressure fluctuation. b) Plots of frequency-domain pressure fluctuation.

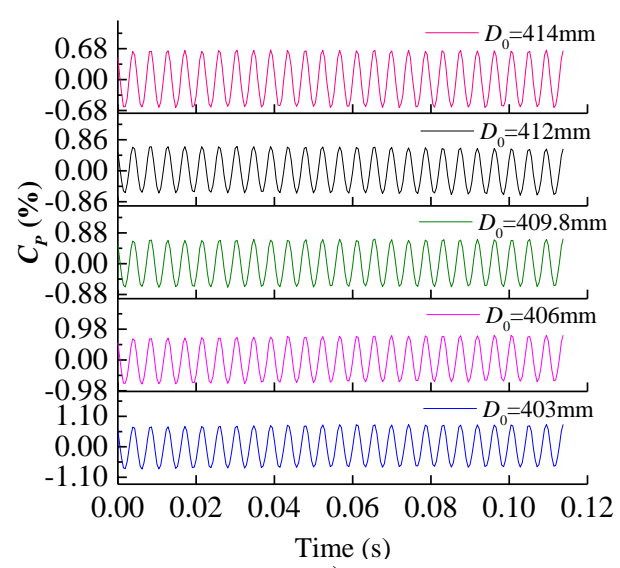

a)

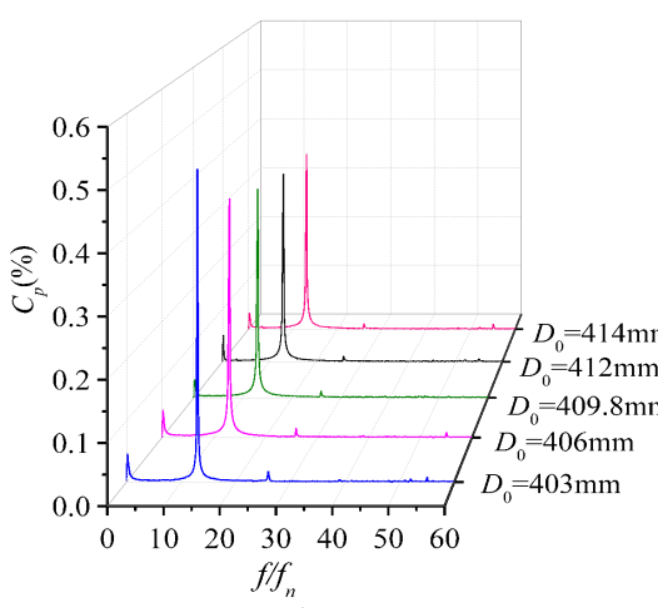

b)

Fig. 11. Plots of time-domain and frequency-domain pressure fluctuation of monitoring points GV12: a) Plots of time-domain pressure fluctuation. b) Plots of frequency-domain pressure fluctuation.

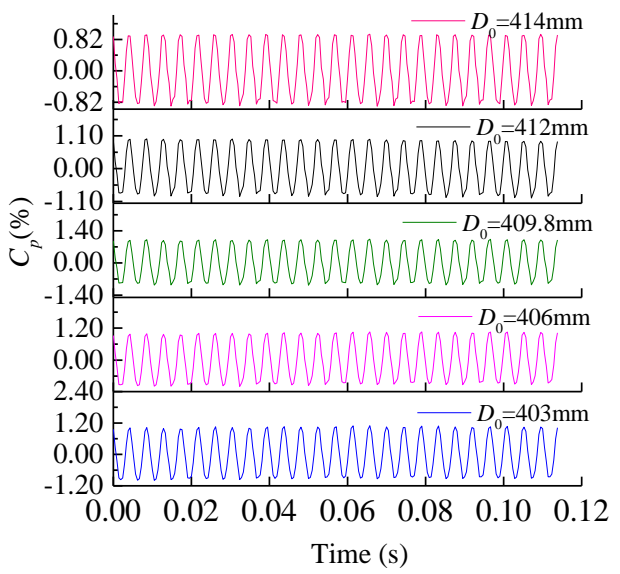

a)

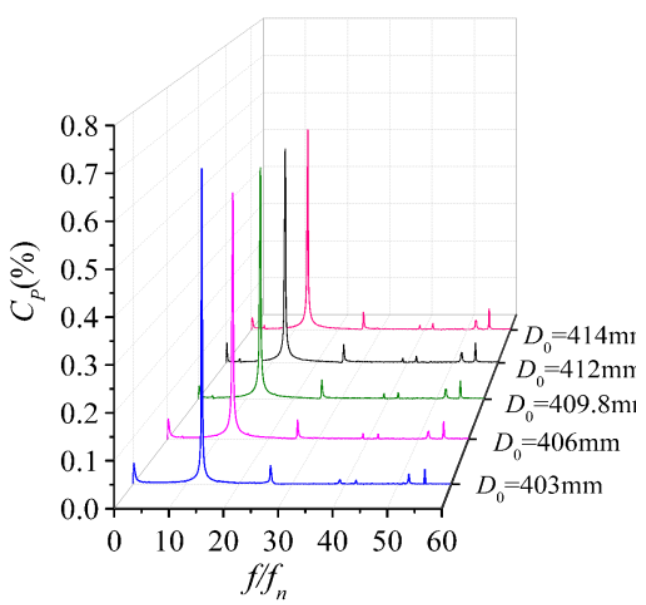

b)

Fig. 12. Plots of time-domain and frequency-domain pressure fluctuation of monitoring points GV13: a) Plots of time-domain pressure fluctuation. b) Plots of frequency-domain pressure fluctuation. 


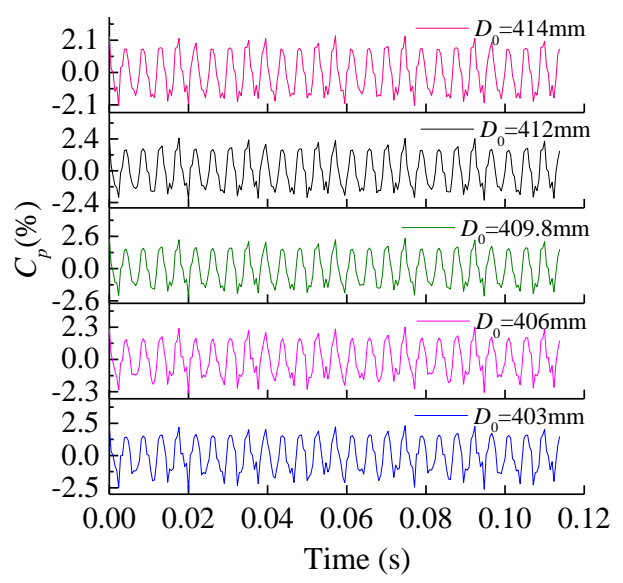

a)

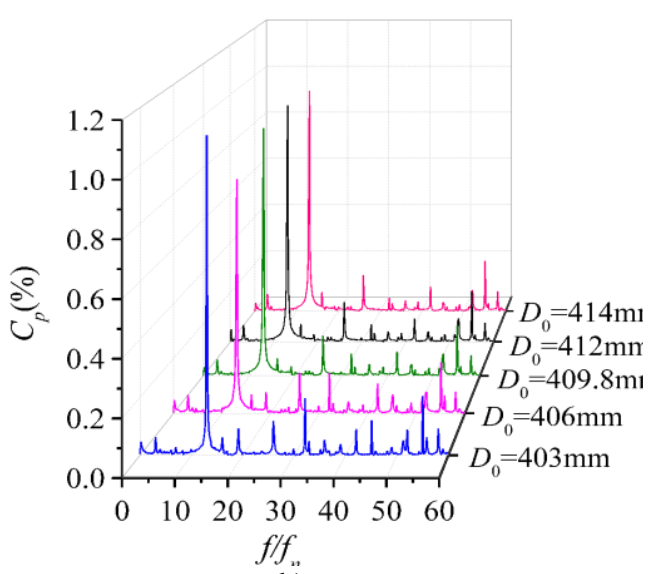

b)

Fig. 13. Plots of time-domain and frequency-domain pressure fluctuation of monitoring points GV14: a) Plots of time-domain pressure fluctuation. b) Plots of frequency-domain pressure fluctuation.

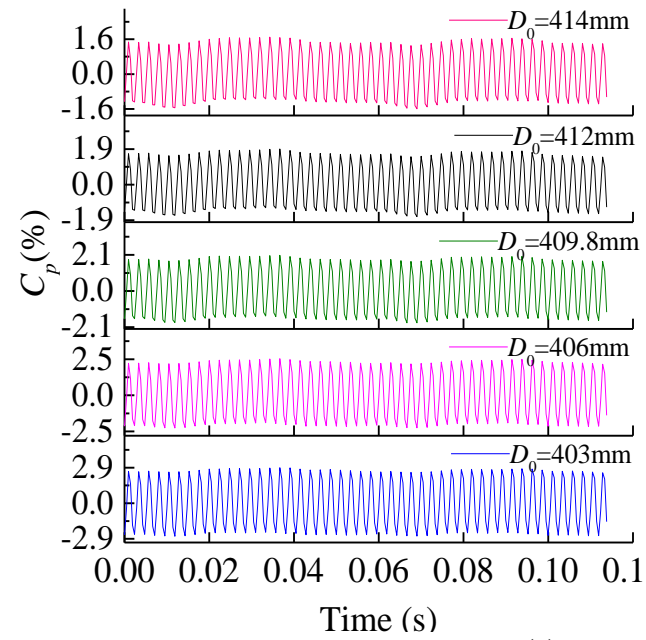

(a)

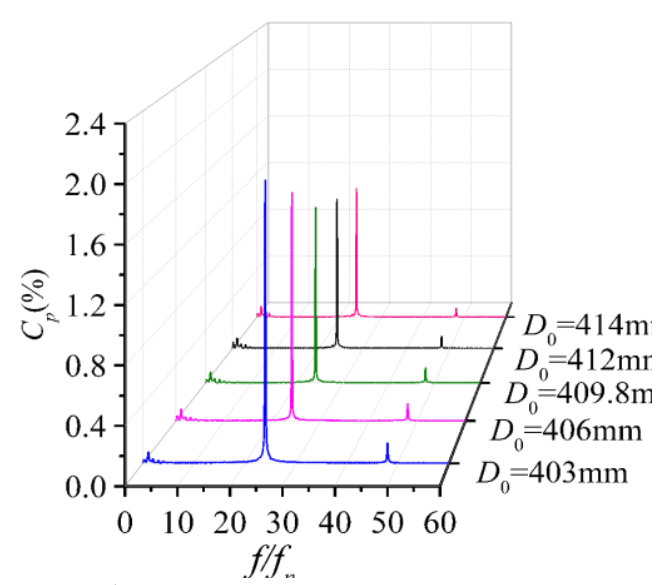

b)

Fig. 14. Plots of time-domain and frequency-domain pressure fluctuation of monitoring points GV15: a) Plots of time-domain pressure fluctuation. b) Plots of frequency-domain pressure fluctuation.

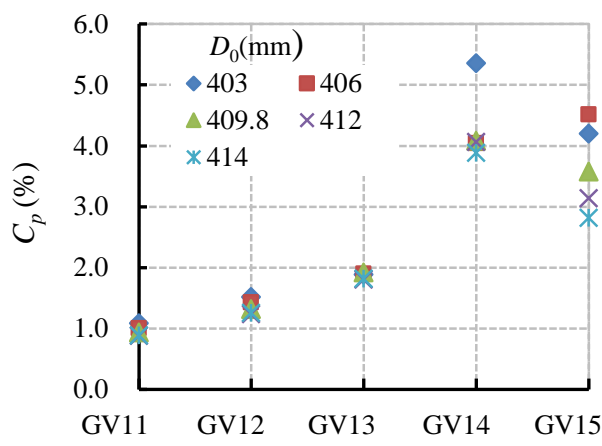

Fig. 15. Peak of pressure fluctuation at monitoring points in vaneless zone.

In the vaneless zone between the guide vanes and the runner, the pressure fluctuation of the vane passing frequency was monitored, and reflects the superposition result of the uneven velocity field and pressure of the guide vanes and those of the inlet of the runner. In this study, the distance between the guide vanes and the runner was changed by altering the guide vane pitch circle diameter, thus the rotorstator interaction between the runner and the guide vane flow field was affected. Figure 16 shows the changing laws of the main frequency amplitude of the pressure fluctuations at monitoring points GV11GV15 under five diameters of the guide vanes distribution circle. As shown in Fig. 16, under the same guide vane pitch circle diameter, the changing laws of the pressure fluctuation at the five monitoring points remained the same, the pressure fluctuation amplitude of GV11 near the top cover was the smallest and that of GV15 near the bottom ring was the largest, and the amplitude of the pressure fluctuation increased gradually from the top cover to the bottom ring. $D_{0}$ increased from $403 \mathrm{~mm}$ to $414 \mathrm{~mm}$, and the pressure fluctuation amplitude of the five monitoring points gradually decreased, among which the decreasing amplitude of the pressure fluctuation at monitoring point GV15 was the greatest, by $0.85 \%(\triangle C p=0.85 \%)$, and that of monitoring point GV13 was the slightest, by $0.15 \%$ 
( $\triangle C p=0.15 \%$ ). Therefore, it could be concluded that the distance between the guide vanes and the runner increased, the rotor-stator interaction between the guide vanes domain and the runner domain decreased, and the pressure fluctuation of the vaneless zone between the guide vanes and the runner was weakened.

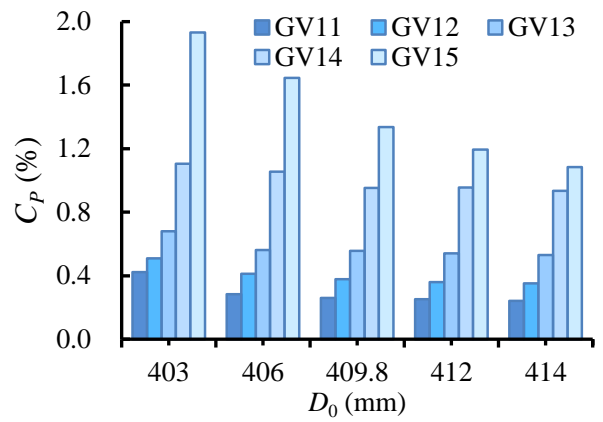

Fig. 16. Pressure fluctuation amplitude in different schemes.

The probability that the value of a random variable falls within a certain region is the integral of the probability density function over that region. The probability density function reflects the probability that the instantaneous amplitude of a signal settles within a certain specified range, and it can be used for characterizing the degree of signal turbulence. In this study, the probability density function was applied to obtain the distribution characteristics of the pressure fluctuation signal of the vaneless zone between the runners in the guide vane domain. The higher the peak value of the probability density function curve is, the more energy would be focused. Additionally, smaller variance indicates a lower dispersion degree of the random variable and more stable data. The probability density function is as follows:

$f(x)=\frac{1}{\sqrt{2 \pi} \sigma} e^{\left(-\frac{(x-\mu)^{2}}{2 \sigma^{2}}\right)}$

$\sigma^{2}=\frac{1}{N} \sum_{i=1}^{N}(x-\mu)^{2}$

Where $\sigma$ is sample data standard deviation, $x$ is the sample value, $\mu$ is the arithmetic mean of the sample, $N$ is the number of samples.

Figure 17 indicates the probability density function curve of the pressure fluctuation at monitoring points GV11-GV15 under the original guide vanes circle case. It can be seen from the Fig. that the peak of the probability density function curve of the pressure fluctuation signal at GV11, which was near the top cover, was relatively high and was distributed in a narrow area with a distribution interval of $5 \mathrm{kPa}$, which indicated a fine high type. The peak of the probability density function curve of the pressure fluctuation peak signal at the monitoring point decreased gradually along the top cover to the bottom ring of the guide vanes, and the distribution range was widened. The peak of the probability density curve of the pressure fluctuation signal at monitoring point GV15 was the lowest, with the widest distribution range of approximately $20 \mathrm{kPa}$. This means that the closer the water flow is to the bottom ring and the smaller the distance is between the guide vanes and the runner inlet, the stronger the rotor-stator interaction of the water flow in the vane domain and the runner domain will be. And the tangential velocity component of the water flow will become higher while, the pressure fluctuation more disordered. Monitoring point GV13 is taken as an example to discuss the influence of different guide vanes on the pressure fluctuation probability density of the monitoring point, as shown in Fig. 18. It can be seen from Fig. 18 that when $D_{0}=403 \mathrm{~mm}$, the peak of the probability density curve of the pressure fluctuation at monitoring point GV13 was the lowest, and the peak value of the curve increased gradually as $D_{0}$ increased. When $D_{0}=414 \mathrm{~mm}$, the peak of the probability density curve of the pressure fluctuation at monitoring point GV13 was the highest and the peak increased by $7.3 \%$ when compared to $D_{0}=403$ $\mathrm{mm}$. As $D_{0}$ increased, the pressure fluctuation energy at monitoring point GV13 gradually concentrated, and the pressure fluctuation signal gradually stabilized.

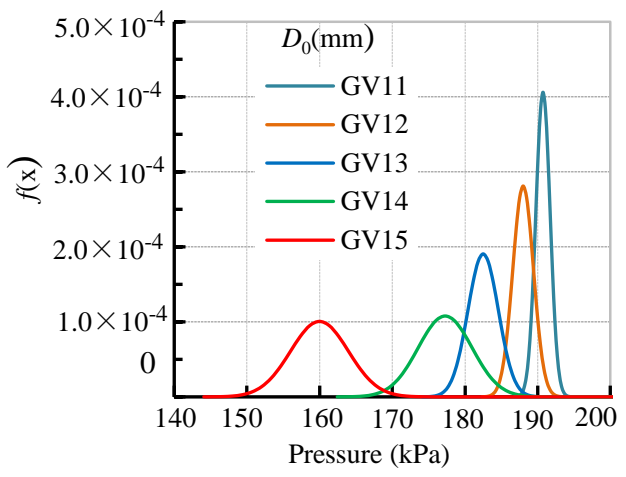

Fig. 17. Pressure fluctuation probability density curve of monitoring points GV11-15 under design flow conditions when $D_{0}=409.8 \mathrm{~mm}$.

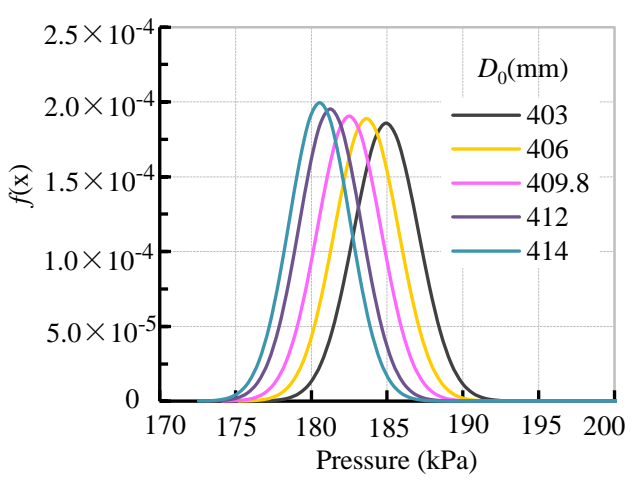

Fig. 18. Pressure fluctuation probability density curve of monitoring point GV13 under design flow conditions but with five schemes when $D_{0}=409.8 \mathrm{~mm}$. 


\section{CONClusions}

Taking a Francis turbine in a power station as the study object, under the condition that the parameters of other flow passage components were constant, five modification schemes were put forward for the guide vane pitch circle diameter. The influence of changes in the guide vane pitch circle diameter on the performance of the Francis turbine, as well as the pressure fluctuation in the vaneless zone between the guide vanes and the runner when the turbine was operating under the design conditions, was analyzed in detail through a numerical simulation of whole passage flow. The conclusions are as follows:

(1) When $D_{0} / D_{1}$ increased from 1.119 to 1.15 , and the turbine efficiency and output showed a monotonically increasing trend, with the efficiency increased by 0.17 percentage point and output increased by $3.91 \mathrm{kw}$.

(2) The pressure fluctuation in the vaneless zone between the guide vanes and the runner is mainly caused by the rotor-stator interaction between the guide vanes and the runner. The pressure fluctuation frequency of the monitoring point in bladed area is mainly composed of the passing frequency of the runner.

(3) From the top cover to the bottom ring, the pressure fluctuation amplitude at monitoring points in the same axial direction increases gradually.

(4) When the unit operates under design conditions, the rotor-stator interaction between the guide vanes and the runner flow field is weakened, and the pressure fluctuation amplitude in the vaneless zone between the guide vanes and the runner is reduced as the guide vane pitch circle diameter is increased, and thus the stability of unit operation could be improved.

In this study, the effect of changes in the guide vane pitch circle diameter on the pressure fluctuation in the vaneless zone of a turbine operating under design conditions is discussed. In future studies, the pressure fluctuation in the vaneless zone of a turbine operating under off-design conditions will be analyzed and discussed, and the influence of the changes in the guide vane pitch circle diameter on the pressure fluctuation in the runner flow field will be further discussed.

\section{ACKNOWLEDGMENTS}

The authors would like to express their gratitude to the respected editor and anonymous reviewers for their useful comments and precious time spent on this paper. This work was supported by "the scientific research Foundation of National Natural Science Foundation of China-Outstanding Youth Foundation" (Grant No. 51622906) and "Shaanxi water conservancy science and technology project" (Project No.: 2019slkj-10).

\section{REFERENCES}

Anup, K. C., B. Thapa and Y. H. Lee (2014). Transient numerical analysis of rotor-stator interaction in a Francis turbine. Renewable Energy 65, 227-235.

Bellendir, E. N., Y. D. Semenov and V. G. Shtengel (2011). First results of inspection of structural components at the Sayano-Shushenskaya HPP after the failure of 17 August 2009. Power Technology and Engineering 44(5), 335-341.

Campobasso, M. S., M. Yan, A. BonFig.lioli, F. A. Gigante, L. Ferrari, F. Balduzzi and A. Bianchini (2018). Low-speed preconditioning for strongly coupled integration of Reynoldsaveraged Navier-Stokes equations and twoequation turbulence models. Aerospace Science and Technology 77, 286-298.

Ciocan, G. D., M. S. Iliescu, T. C. Vu, B. Nennemann and F. Avellan (2007). Experimental Study and Numerical Simulation of the FLINDT Draft Tube Rotating Vortex. Journal of Fluids Enineering 129(2), 146-158.

Gavrilov, A. A., A. V. Sentyabov, A. A. Dekterev and K. Hanjalic (2016). Vortical structures and pressure pulsations in draft tube of a Francis-99 turbine at part load: RANS and hybrid RANS/LES analysis. International Journal of Heat and Fluid Flow 63, 158-171.

Giosio, D. R., A. D. Henderson, J. M. Walker and P. A Brandner (2017). Rapid reserve generation from a Francis turbine for system frequency control. Energies 10(4), 496.

Han, L., H. J. Wang, Y. H. Gao, D. Y. Li, R. Z. Gong and X. Z. Wei (2017). Dynamic simulation in guide vane opening process of a pump-turbine in pump mode. Journal of Applied Fluid Mechanics 10(5), 1441-1449.

Iovănel, R. G., G. Dunca and M. J. Cervantes (2019). Study on the accuracy of rans modelling of the turbulent flow developed in a kaplan turbine operated at BEP. Part 2 - pressure fluctuations. Journal of Applied Fluid Mechanics 12(5), 1463-1473.

Koirala, R., B. Thapa, H. P. Neopane, B. S. Zhu (2017). A review on flow and sediment erosion in guide vanes of Francis turbines. Renewable and Sustainable Energy Reviews 75, 10541065 .

Koirala, R., B. Zhu and H. P. Neopane (2016). Effect of guide vane clearance gap on Francis turbine performance. Energies 9(4), 275.

Lain, S., M. A. Taborda and O. D. Lopez (2018). Numerical study of the effect of winglets on the performance of a straight blade darrieus water turbine. Energies 11(2): 297.

Li. Q. F., R. N. Li, H. Quan and W. Han (2011, December). Solid-liquid Two-phase Flow numerical simulation around guide vanes of mixed-flow water turbine. International 
Z. J. Wu et al. / JAFM, Vol. 13, No. 5, pp. 1349-1361, 2020.

Conference on Advances in Computational Modeling and Simulation, Kunming, China.

Menter, F. R. (1994). Two-equation eddy-viscosity turbulence models for engineering applications. AIAA Journal 32 (8), 269-289.

Minakov, A. V., D. V. Platonov, A. A. Dekterev, A. V. Sentyabov and A. V. Zakharov (2015). The numerical simulation of low frequency pressure pulsations in the high-head Francis turbine. Computers \& Fluids 111, 197-205.

Muller, A., A. Bullani, M. Dreyer, S. Roth, A. Favrel, C. Landry and F. Avellan (2012, August). Interaction of a pulsating vortex rope with the local velocity field in a Francis turbine draft tube. 26th IAHR Symposium on Hydraulic Machinery and Systems, Beijing, China.

Peltier, R., A. Boyko, S. Popov and N. Krajisnik (2010). Investigating the Sayano-Shushenskaya Hydro Power Plant Disaster. Power 154 (12), 48.

Pereiras, B., P. Valdez and F. Castro (2014). Numerical analysis of a unidirectional axial turbine for twin turbine conFig.uration. Applied Ocean Research 47, 1-8.

Romero-Gomez, P., S. F. Harding, M. C. Richmond (2017). The effects of sampling location and turbulence on discharge estimates in short converging turbine intakes. Engineering Applications of Computational Fluid Mechanic
11(1), 513-525.

Staubli, T.; F. Senn and M. Sallaberger (2008, October). Instability of Pump-TurbinesDuring Start-Up in Turbine Mode. Hydro 2008, Ljubljana, Slovenia.

Thapa, B. S., C. Trivedi and O. G. Dahlhaug (2016). Design and development of guide vane cascade for a low speed number Francis turbine. Journal of Hydrodynamics, Ser. B 28(4), 676-689.

Trivedi, C., E. Agnalt and O. G. Dahlhaug (2017). Investigations of unsteady pressure loading in a Francis turbine during variable speed operation. Renewable Energy 113, 397-410.

Trivedi, C., M. J. Cervantes and B. K. Gandhi (2016). Investigation of a high head Francis turbine at runaway operating conditions. Energies 9,149.

Tyagi, R. K. (2015). The effect of an angle on the impact and flow quantity on output power of an impulse water wheel model. Journal of Energy in Southern Africa 26(3), 100-104.

Yan, J., J. Koutnik, U. Seidel and B. Hubner (2010, September). Compressible simulation of rotorstator interaction in pump-turbines. 25th IAHR Symposium on Hydraulic Machinery and Systems. Timisoara, Romania. 